\title{
Application of the Acoustic Emission Method for Diagnosis of On-Load Tap Changer
}

\author{
Henryk MAJCHRZAK, Andrzej CICHOŃ, Sebastian BORUCKI \\ Faculty of Electrical Engineering, Automatic Control and Computer Science \\ Opole University of Technology \\ Prószkowska 76, 45-758 Opole, Poland; e-mail: a.cichon@po.opole.pl \\ (received February 12, 2016; accepted October 10, 2016)
}

\begin{abstract}
This paper provides an example of the application of the acoustic emission (AE) method for the diagnosis of technical conditions of a three-phase on-load tap-changer (OLTC) G III type. The measurements were performed for an amount of 10 items of OLTCs, installed in power transformers with a capacity of 250 MVA. The study was conducted in two different OLTC operating conditions during the tapping process: under load and free running conditions. The analysis of the measurement results was made in both time domain and time-frequency domain. The description of the AE signals generated by the OLTC in the time domain was performed using the analysis of waveforms and determined characteristic times. Within the time-frequency domain the measured signals were described by short-time Fourier transform spectrograms.
\end{abstract}

Keywords: acoustic emission; partial discharge; transformer diagnostics.

\section{Introduction}

Power transformers are one of the most important items in the national electrical system (BŁACHOWICZ et al., 2016; FARAG et al., 1999; GARCIA-COLON et al., 2004; LIN et al., 2005). A properly conducted transformer maintenance is essential to optimize the total power system maintenance costs, and in particular, to ensure the continuity of the electricity supply service to consumers (LOPATKIEWICZ et al., 2012; MENON et al., 2001; Witos, GACEK, 2008). A failure to comply with the $n-1$ secure condition, may lead to the breach of the terms and conditions of the transmission contracts binding transmission and distribution system operators as well as electricity market participants. These practices generally have financial consequences. Therefore, transformer operations require continuous monitoring of the diagnostic indicators that describe the current conditions of these devices (DENG et al., 2001; Grossmann, Feser, 2001; NAderi et al., 2007; SHARKAWY et al., 2005; WANG et al., 2006; ZHANG et al., 2007).

On-load tap-changers (OLTCs) are one of the main causes of failures in power transformers. The causes of OLTC failures can be divided into three major categories: mechanical system failures (mainly related to the transmission system), damages produced in the main current path caused by wear or damage of the contacts, and problems in the insulation system (FoATA et al., 2000; Rivas et al., 2009; Filho, DE AlmeIDA, 2008; WANG et al., 2008). Figure 1 presents basic types of OLTC failures.

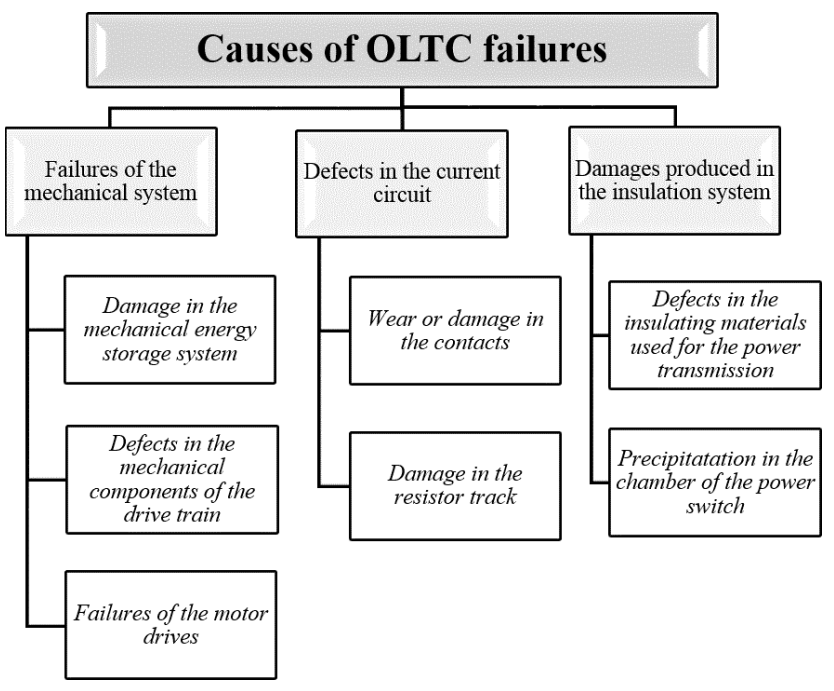

Fig. 1. Types of OLTC failures. 
Despite the fact that the OLTCs are components of the transformer and that they have a relatively high failure rate, for many years, their technical condition analysis was carried out on the basis of an internal inspection and a visual observation of the power switch contact deterioration. In order to determine the level of the power switch contact deterioration in a noninvasive manner, and to check clamp fittings of the current circuit, an oscillographic method was developed. Nowadays, this method is the primary diagnostic method used under normal operating conditions. An additional parameter which constitutes an important complement to oscillographic tests is the winding resistance measurement, which allows comprehensive diagnosis of current transformer circuits, being mainly the contacts of the selector.

The results derived from the winding resistance measurements and the oscillographic tests provided detailed information concerning the OLTC technical conditions. The biggest limitation of the discussed diagnostic method is the possibility to perform measurements only off-line, that is, when the power transformer is disconnected from the voltage.

There are also systems dedicated only to selected types of OLTCs, which makes it possible to conduct diagnostics on-line. An example of a comprehensive approach to the analysed issue is the OLTC monitoring system, presented in (CICHOŃ et al., 2012; 2014; 2015; Foata et al., 2000; Rivas et al., 2009; Filho, De Almeida, 2008; Wang et al., 2008). Currently, the main direction of further research studies on this subject matter is the development of effective and noninvasive methods. These systems aim to determine the technical conditions of all types of OLTCs, which will allow their on-line diagnosis.

The OLTC switching process refers to the generation of acoustic signals, which allows to use the AE methods to diagnose their technical conditions. The AE method is commonly used for the diagnosis of technical equipment (Bolin, 1979; Pollock, Stephens, 1971). During the switching process, the AE signals can be generated by switching the mechanical system, contacting movements or with electrical discharges. For most types of OLTCs, the working environment is oil insulation, through which the arising acoustic pressure wave propagates and reaches the metal tank. Therefore, there is a possibility to record AE signals by using a piezoelectric transducer attached to the external part of the tank. These obtained AE signals provide useful information which can successfully describe the operation of both the power switch and the selector (Cichoń et al., 2012; 2014; 2015). The acoustic signal generated during the OLTC switching cycle contains information about two diagnostic areas of the power switch. The first area is related to the assessment of the mechanical conditions of contacts and the transmission system. The second area is related to the switch diag- nostics in order to identify the electrical nature of the defects, commonly known as detection of electric discharges with increased intensity.

Each type of OLTC generates an AE signal that has its own characteristics. It is possible to determine the AE signal characteristics describing the most common OLTC defects. By using AE signals recorded for new OLTCs and having a base of acoustic descriptors corresponding to specific types of defects, it is possible to carry out an effective assessment of the test switch technical conditions by making a comparison between the AE signals and the reference signals, forming the so-called 'fingerprint'.

\section{Characteristics of the analysed OLTCs}

During the research works, whose results are presented in this article, an acoustic signal recording was performed at 10 OLTC units of G III type. The test contacts were attached to the tank bottom of the power transformers with a capacity of 250 MVA, filled with insulation oil. The analysed OLTCs were designed with separate selectors and power switches. They also possess a complex resistor circuit, which allows for a better on-load voltage control of the transformer in three phase circuits at load currents of up to $3 \times 1600$ A. Figure 2 shows a general view of the transformer and the OLTC compartment (Fig. 2a), and a general view of the of G III type power switch.

a)
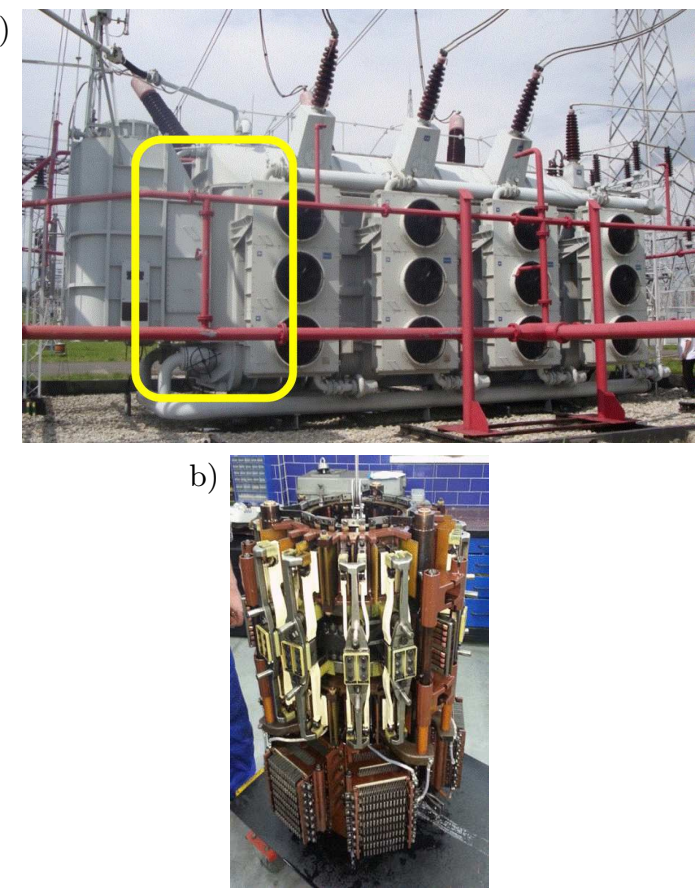

Fig. 2. General view of the transformer with an OLTC compartment - marked by white (a) and the G III type power switch (b).

From the ten initial OLTCs under study at the first research stage, two switches were identified. One 
(OLTC No. 1) was in a good technical condition, and the second one (OLTC No. 2), due to long-term operation and a large number of switching cycles, was suspected to be damaged. On the one hand, a general overhaul procedure of the power switch was conducted in the OLTC No. 1, and the recorded acoustic signals for this device resulted to be the so-called 'fingerprints'(with a total amount of ten OLTCs). On the other hand, the OLTC No. 2 was characterised by a relatively long operating life, and the results of its electrical safety testing indicated the possibility of an excessive wear of the contacts in the head of the power switch.

\section{Characteristics of the measurement system}

The AE signals were recorded using a piezoelectric pin transducer WD type AH17 with wide frequency range attached on the top of the transformer, near the power switch. The sampling frequency was $400 \mathrm{kHz}$ and 2000000 samples were recorded. This allowed for an AE signal measurement within $5 \mathrm{~s}$, while the signal gain was $20 \mathrm{~dB}$.

The received AE signals by the WD AH17 piezoelectric sensor were amplified in the preamplifier system (type $2 / 4 / 6$ ). From the preamplifier, the AE measuring signal was transmitted to an amplifying circuit (type AE2A). After that, a band-pass filter with cut-off frequencies 1 and $200 \mathrm{kHz}$ was applied to the system (Fig. 3).

a)

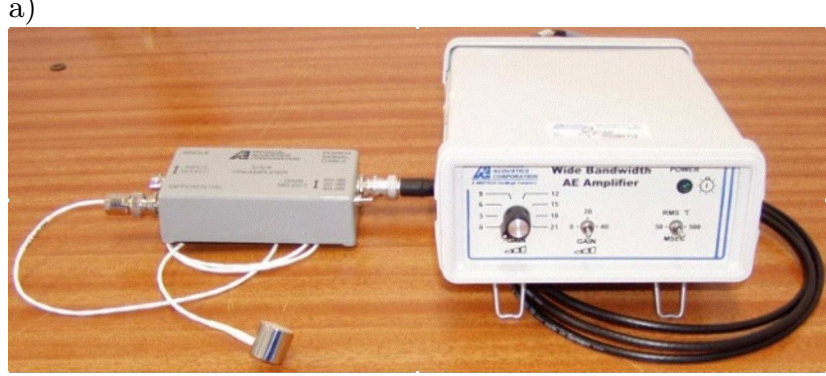

b)

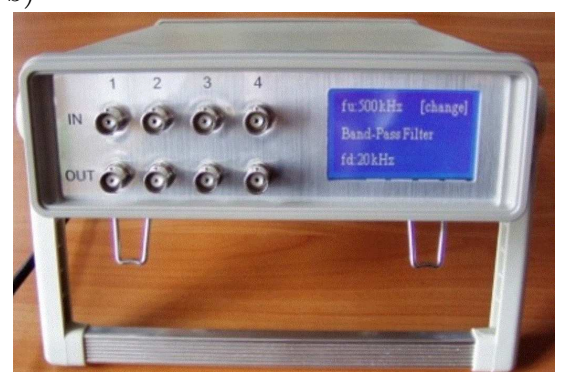

Fig. 3. General view of the measurement system: a) broadband transducer WD type AH 17 with preamplifiers and an amplifier, b) band-pass filter.

The AE signal waveforms generated within the tapping cycle of the analysed OLTCs were registered on the discs of a measuring PC using a four-channel measurement card - type CH 3160 (Fig. 4).

a)

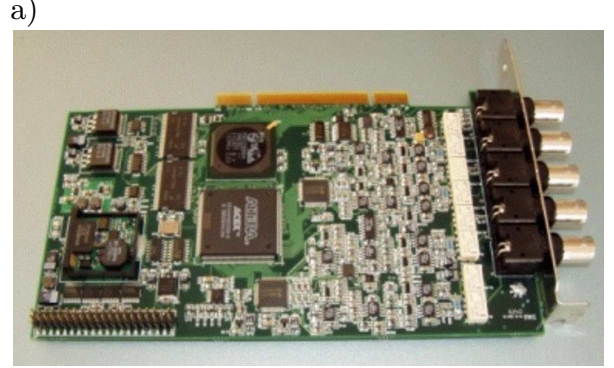

b)

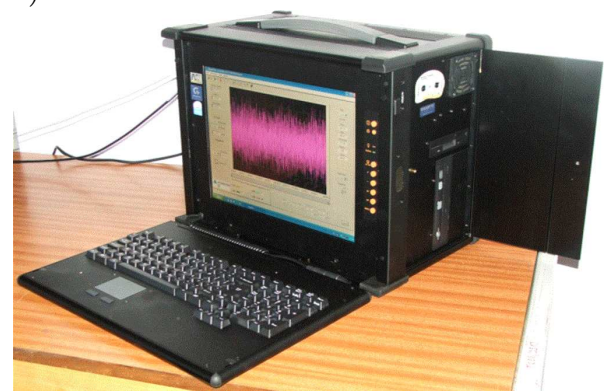

Fig. 4. General view of the applied measurement card (a) and the measuring industrial PC (b).

\section{Methodology for signal measurements and analysis}

The recording of the AE signals generated during the OLTC switching cycle was performed under two different transformer operating conditions: under load and under free running.

The AE signal analysis recorded during OLTC operation was performed in time and time-frequency domains. The assessment of signals in the time-domain was based on the determination of the so-called characteristic times. The three characteristic times that characterize the typical distance between structures are: the first and second $-T_{1}$, the second and third $-T_{2}$, and the first and third $-T_{c}$.

The way of determining the characteristic times is presented in Fig. 5.

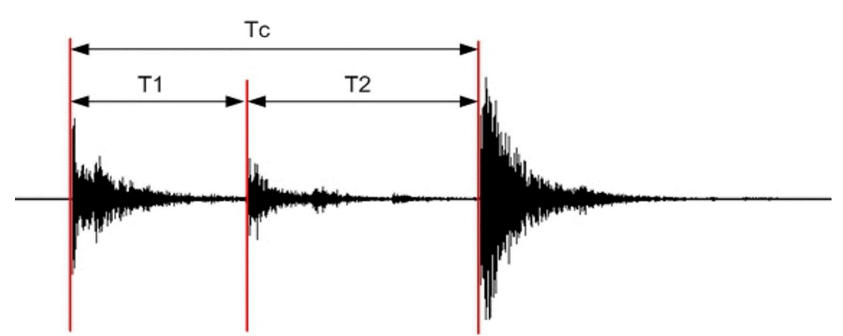

Fig. 5. The way of determining characteristic times of the AE signals generated by OLTC.

The time-frequency analysis of the AE signals generated by the OLTC was performed in the environ- 
ment of the Matlab application, based on a short-time Fourier transform (STFT).

During the designation of the 'fingerprint' for characteristic time values of acoustic signals generated by the OLTC - type G III, a statistical analysis of the data obtained was carried out. Table number 1 is a summary of the characteristic time values of the measured switch, which were designated based on the measurements of the AE signals generated during the switching process of the OLTC head. The time values presented in the table below were considered as standard and characteristic for the OLTC type G III test - without any defects of mechanical and electrical character.

Table 1. Ranges of the characteristic times of the power switch type G III adopted as standard in OLTCs operating without failure.

\begin{tabular}{|c|c|c|}
\hline$T_{1}[\mathrm{~ms}]$ & $T_{2}[\mathrm{~ms}]$ & $T_{c}[\mathrm{~ms}]$ \\
\hline $32.0-36.0$ & $22.0-26.0$ & $50-60.0$ \\
\hline
\end{tabular}

\section{Analysis of the results}

Figure 6 presents different examples of AE signal waveforms generated in the tested OLTCs which were

a)

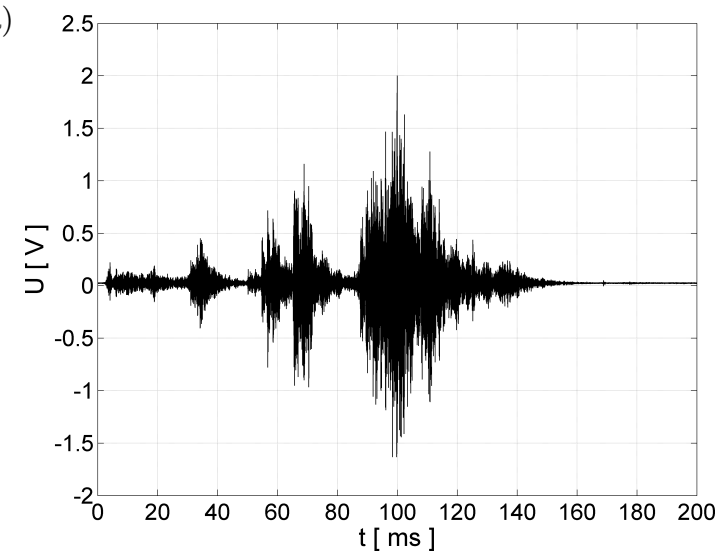

b)

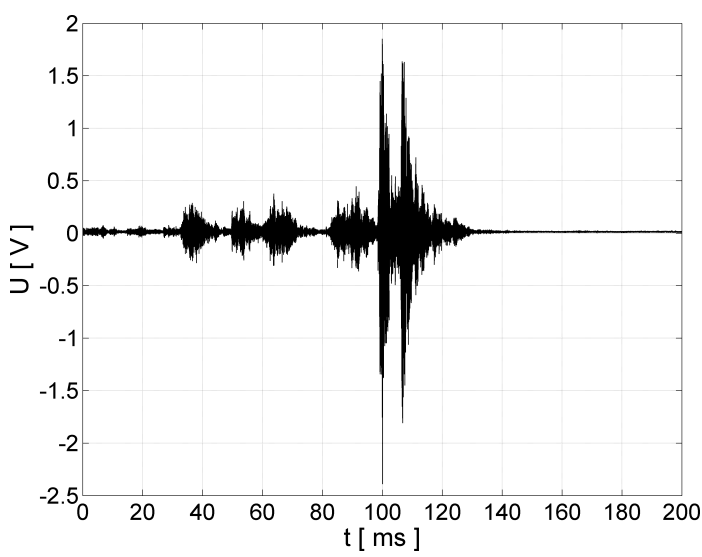

Fig. 6. Exemplary waveform of the AE signals generated by OLTC within the switching cycle under load running conditions: a) OLTC No.1, b) OLTC No. 2. recorded within the switching cycle under load. Figure 7 shows oscillograms obtained during the analysis of signals recorded at the moment of switching OLTCs under free running conditions with a switched off transformer. The 'a' symbol refers to the waveforms of the AE signals generated under the switching of OLTC head No. 1, whereas the ' $b$ ' symbol states for OLTC head No. 2.
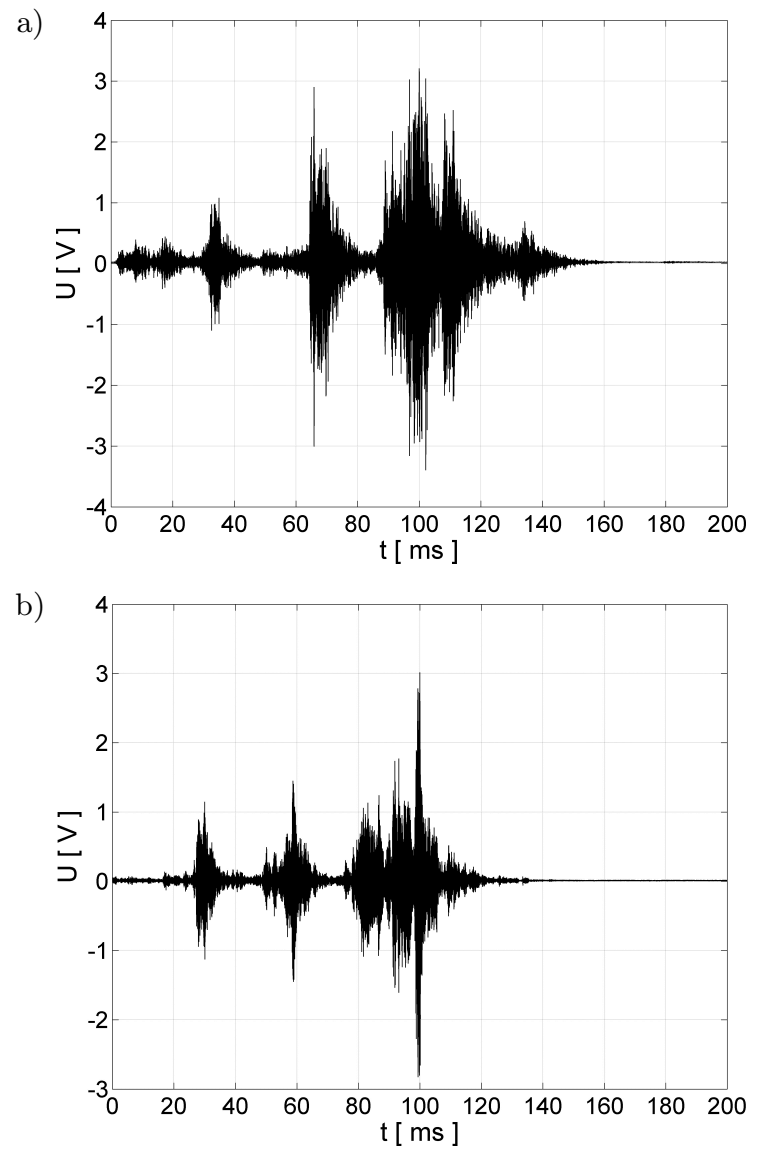

Fig. 7. Exemplary waveforms of the AE signals generated by the analysed OLTCs in the tapping cycle under free

running conditions: a) OLTC No. 1, b) OLTC No. 2.

Table 2 provides averaged values of No. 1 and No. 2 OLTC time characteristics, which were determined during the AE signal processing, and registered during switching them on and off under load and free running conditions. The table below shows the average time characteristics based on a sample of more

Table 2. Averaged characteristic times determined for the AE signals generated by the analysed OLTCs.

\begin{tabular}{|c|c|c|c|c|}
\hline OLTC name & OLTC running mode & $\begin{array}{c}T_{1} \\
{[\mathrm{~ms}]}\end{array}$ & $\begin{array}{c}T_{2} \\
{[\mathrm{~ms}]}\end{array}$ & $\begin{array}{c}T_{c} \\
{[\mathrm{~ms}]}\end{array}$ \\
\hline \multirow{2}{*}{ OLTC No. 1 } & load running & 34.27 & 23.08 & 57.35 \\
\cline { 2 - 5 } & free running & 33.00 & 24.85 & 58.34 \\
\hline \multirow{2}{*}{ OLTC No. 2 } & load running & 31.02 & 21.03 & 52.05 \\
\cline { 2 - 5 } & free running & 28.97 & 21.56 & 51.53 \\
\hline
\end{tabular}


than 100 measurement cycles of each tested OLTC. The maximum uncertainty of the mentioned characteristic times was calculated using $t$-Student's distribution, assuming the probability $P=0.95$, did not exceed $\pm 2.1 \mathrm{~ms}$ for each indicator.

Based on the analysis of the waveforms and designated characteristic times of OLTC operating under load and free running condition, it was stated that for OLTC No. 1 all the determined values of characteristic times (Table 2) are within the ranges considered as common references (Table 1). However, for OLTC No. 2, a decrease of $T_{1}, T_{2}$ and $T_{c}$ times (Table 2) was observed, in relation to those adopted by the authors as the so-called common reference intervals (Table 1).

The performed study indicated that the AE signal waveforms generated within OLTC switching cycle under load (Fig. 6) are characterized by four consecutive acoustic events. However, acoustic signals recorded within OLTC operating under free running conditions (Fig. 7) are characterized by only three distinct structures. The presence of an additional acoustic event in the EA signal waveforms measured within OLTC running under load, corresponds to the acoustic wave generated by an electric arc, which ignites between the power-head working contacts during the tap change. The EA signal analysis also showed that the structure and character of time-amplitude acoustic events generated by the OLTC No. 2 (Figs. $6 \mathrm{~b}$ and $7 \mathrm{~b}$ ) have a totally different shape compared to the measured signals for the total examined amount of OLTCs of G III type. This is particularly evident in the last acoustic structure, which in the initial part has a small amplitude, and then two distinct maxima (Figs. $6 \mathrm{~b}$ and $7 \mathrm{~b}$ ).

The AE signal analysis of the examined OLTCs was extended by an analysis carried out in the field of timefrequency domain, determining two-dimensional spectrograms of power density spectra presented in Figs. 8 and 9. The spectrograms shown in Fig. 8 represent images derived from the basis of the EA signal processing, which was recorded during the switching cycle of the OLTC under load. However, Fig. 9 shows the results of the time-frequency analysis of the AE signals measured in the same OLTCs, but under free running conditions. The 'a' symbol indicates the results of the STFT analysis of the AE signals generated in switching conditions. The 'a' symbol refers to OLTCs \#1, whereas the ' $b$ ' symbol refers to OLTCs \#2.

In the presented spectrograms of power density spectra there are visible time-frequency structures with a dominant frequency band within the range 10$100 \mathrm{kHz}$ (Figs. 8 and 9). At the end of the analysed waveform there are structures with a maximum amplitude. On the images of power density spectra, two major frequency bands can be distinguished, in which local maxima occur. The first one is within the frequency range $10-30 \mathrm{kHz}$, and the second one within the range $45-55 \mathrm{kHz}$. The analysis of the test results also proved a)

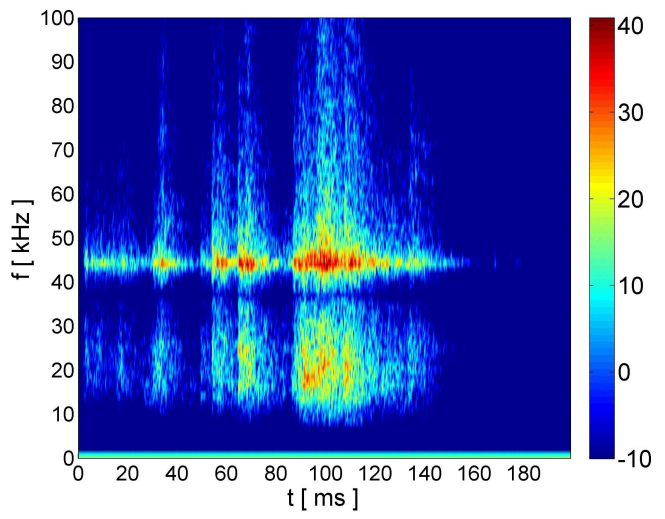

b)

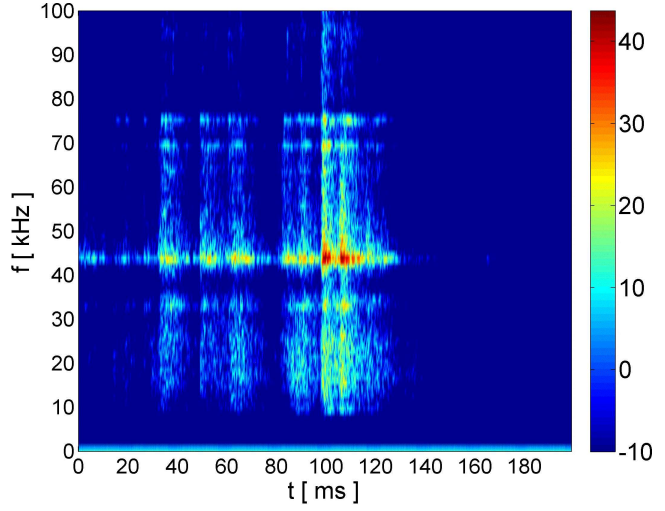

Fig. 8. Exemplary STFT spectrograms of the AE signals generated by OLTC within the switching cycle under load running conditions: a) OLTC No. 1, b) OLTC No. 2.

a)

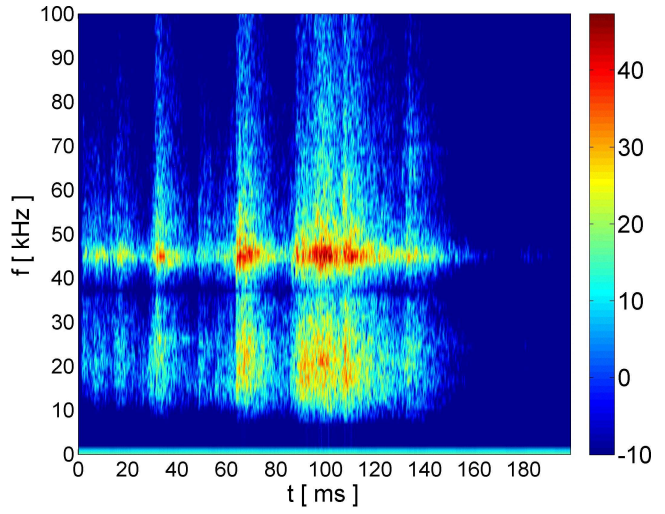

b)

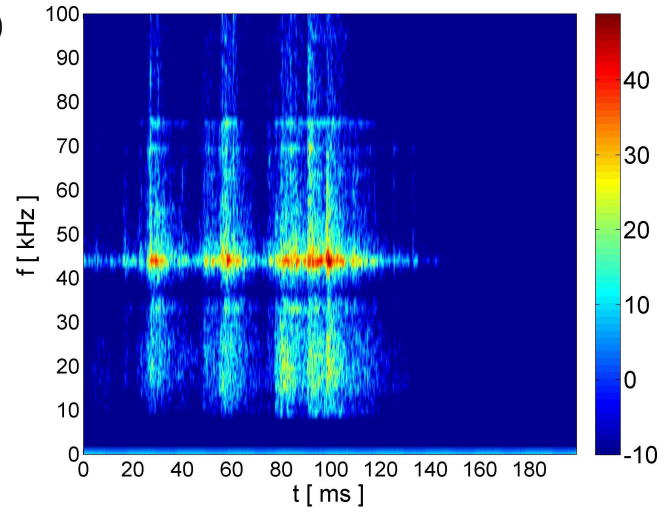

Fig. 9. Exemplary STFT spectrograms of the AE signals generated by OLTC within the free running switching cycle: a) OLTC No. 1, b) OLTC No. 2. 
that the signals corresponding to the work of OLTCs No. 1 have time-frequency wavelet structures with the maximum amplitudes (Figs. 8a and 9a). Based on the STFT analysis of two-dimensional surfaces designated for the AE signals generated by OLTC No. 2 (Figs. 8b and $9 \mathrm{~b}$ ), clear differences in their time-frequency structure can be observed. The OLTC No. 2 shows a distinct layer separation in the last acoustic structure of the analysed AE signal, as well as different location of particular frequency structures in time and other amplitude distribution in the range $10-30 \mathrm{kHz}$ (Figs. $8 \mathrm{~b}$ and $9 \mathrm{~b})$.

The studies performed did not show significant differences in the time-frequency images of AE signals recorded during the tests conducted on the OLTCs operating under load and free running conditions. STFT image structures are characterised by the same participation of dominant frequencies, and also by a convergence of particular time and amplitude distribution. The presence of additional time-frequency structures on spectrograms of power density spectra of AE signals measured at OLTC running under load (Fig. 8) is related to the generation of acoustic waves from electric arc ignition. On the basis of the performed experimental trials, it can be stated that it is possible to apply the AE method for the OLTC technical condition estimation only under one of the two working conditions of the on-load tap changer contacts: under load or free running conditions.

\section{Summing-up}

Based on the results presented in this paper it can be concluded that by using modern digital signal processing methods in the time and time-frequency domains, it is possible to apply the EA method for the OLTC technical condition estimation of construction with a separate selector and power switch, during its normal operation.

The comparison of measurement results for OLTC operating under load and free running conditions allows to formulate analogous conclusions concerning the current technical condition of these devices. The research conducted under this publication showed that from the diagnostic point of view it is sufficient to record and analyse AE signals from OLTCs running under load. Therefore, this statement allows to conduct OLTC periodic diagnostics without needing neither to shut down the transformer nor to exclude the OLTCs from the operation.

On the basis of the conducted measurements of acoustic emission of OLTC No. 1 and the performed analysis of the generated AE signals, it was concluded that in the OLTCs test mechanical and electrical failures did not occur.

Because of the distinct exception of the designated time and time-frequency parameters of AE signals gen- erated by the OLTC No. 2, it was recommended to carry out a further detailed research on this switch using the oscillograms method. In case of obtaining negative results, an inspection should be carried out. The character of the analysed AE signals may indicate the beginning of the No. 2 OLTC contact deterioration process.

Currently, research studies are being performed with the purpose of verifying the diagnostic method for the other types of OLTCs described in the article.

\section{References}

1. BŁachowicz A., Boczar T., Wotzka D. (2016), Application of a mobile system in diagnostics of power capacitors using the acoustic emission method, Insight, 58, 2, 94-100.

2. Bolin L. (1979), A model for estimating the signal from an acoustic emission source, Ultrasonics, 17, 2, $67-70$.

3. Cichoń A., Berger P., Wotzka D. (2015), Selecting Mother Wavelet for Wavelet Analysis of On-Load Tap Changer Acoustic Emission, Acta Physica Polonica A, 128, 310-314.

4. Cichoń A., Borucki S., Wotzka D. (2014), Modeling of Acoustic Emission Signals Generated in On Load Tap Changer, Acta Physica Polonica A, 125, 13961399.

5. Cichoń A., Frącz P., Boczar T., Zmarzly D. (2012), Detection of Defects in On-Load Tap-Changers Using Acoustic Emission Method, Proceedings of International IEEE Symposium on Electrical Insulation, San Juan, 184-188.

6. Deng J., Xiao H., Huo W., Luo M., May R., WANG A., LIU Y. (2001), Optical fiber sensor-based detection of partial discharges in power transformers, Optics \& Laser Technology, 33, 305-311.

7. Farag A.S., Shewhdi M.H., Jin X., Wang C., Cheng T.C., Dong X., Gao S., Jing W., Wang Z. (1999), On-line partial discharge calibration and monitoring for power transformers, Electric Power Systems Research, 50, 47-54.

8. Filho E.F.S., De Almeida L.A.L. (2008), Selforganized classification of on - load tap changers acoustic signature, Proceedings of the IEEE International Conference on Instrumentation and Measurement Technology, Victoria BC, pp. 1051-1056.

9. Foata M., Beauchemin R., Rajotte C. (2000), On line testing of on-load tap changers with a portable acoustic system, Proceedings of the IEEE Conference on Transmission and Distribution Construction, Operation and Live-Line Maintenance, pp. 293-298.

10. Garcia-Colon V.R., Linan-Garcia R., JaCOBO M.A. (2004), On-line condition assessment of high voltage current transformers, Conference Record of the IEEE International Symposium on Electrical Insulation, Indianapolis, pp. 182-185. 
11. Grossman E, Feser K. (2001), Online PDmonitoring on transformers using AE techniques, Proceedings of International Conference on APTADM, Wrocław, pp. 264-268.

12. Lin D., Jiang L., Li F., Zhu D., Tan K., Wu C., Jin X., Wang C., Cheng T.C. (2005), On-line partial discharge monitoring and diagnostic system for power transformer, Tsinghua Science \& Technology, 10, 598604 .

13. Lopatkiewicz R., NAdolny Z., Przybylek P. (2012), The Influence of Water Content on Thermal Conductivity of Paper Used as Transformer Windings Insulation, Proceedings of 10th IEEE International Conference on the Properties and Applications of Dielectric Materials, Bangalore, pp. 1-4.

14. Menon R., Kolambekar S., Buch N.J., RaMAMOORTY M. (2001), Correlation of acoustic emission method and electrical method for detection of partial discharges in transformer, Proceedings of 7th IEEE International Conference on Solid Dielectrics, Eindhoven, pp. 299-302.

15. NAderi M.S., Vakilian M., Blackburn T.R., Phung B.T., NAsiri A. (2007), A hybrid transformer model for determination of partial discharge location in transformer winding, IEEE Transactions on Dielectrics and Electrical Insulation, 14, 436-443.

16. Pollock R.W.B., Stephens A.A. (1971), Waveform and frequency spectra of acoustic emissions, The Journal of Acoustical Society of America, 49, 110-114.
17. Rivas E., Burgos J.C., Garcia-Prada J.C. (2009), Condition Assessment of Power OLTC by Vibration Analysis Using Wavelet Transform, IEEE Transactions on Power Delivery, 24, 687-694.

18. Sharkawy R.M., Fakhry S., Anis H., GhazaLY H.A. (2005), Particle detection in oil using acoustic and electrical based techniques in correlation with an inference method, Proceedings of Conference on Instrumentation and Measurement Technology, Ottawa, pp. 1080-1085.

19. Wang P., Li B., Roman H.T., Russo O.L., Chin K., FARMER K.R. (2006), Acousto-optical PD detection for transformers, IEEE Transactions on Power Delivery, 21, 1068-1073.

20. Wang Y., Zhou J., Chen W., Du L., Chen R., Zhang L., Song W. (2008), Study of an Assessment Method for the Reliability of Tap-changers in Power Transformer Based on Fault-Tree Analysis, Proceedings of the International Conference on High Voltage Engineering and Application, Chongqing, pp. 604-608.

21. Witos F., GACEK W. (2008), Application of the joint electro-acoustic method for $P D$ investigation within a power transformer, The European Physical Journal - Special Topics, 154, 239-247.

22. Zhang H., Blackburn T.R., Phung B.T., Sen D. (2007), A novel wavelet transform technique for on-line partial discharge measurements: Part 2: On-site noise rejection application, IEEE Transactions on Dielectrics and Electrical Insulation, 14, 15-22. 